\title{
On the initial-value problem in a rotating circular cylinder
}

\author{
KEKE ZHANG ${ }^{1}$ AND XINHAO LIAO ${ }^{2}$ \\ ${ }^{1}$ Department of Mathematical Sciences, University of Exeter, Exeter, EX4 4QE, UK \\ ${ }^{2}$ Shanghai Astronomical Observatory, Chinese Academy of Sciences, Shanghai 200030, China
}

(Received 9 May 2007 and in revised form 5 June 2008)

The initial-value problem in rapidly rotating circular cylinders is revisited. Four different but related analyses are carried out: (i) we derive a modified asymptotic expression for the viscous decay factors valid for the inertial modes of a broad range of frequencies that are required for an asymptotic solution of the initial value problem at an arbitrarily small but fixed Ekman number; (ii) we perform a fully numerical analysis to estimate the viscous decay factors, showing satisfactory quantitative agreement between the modified asymptotic expression and the fuller numerics; (iii) we derive a modified time-dependent asymptotic solution of the initial value problem valid for an arbitrarily small but fixed Ekman number and (iv) we perform fully numerical simulations for the initial value problem at a small Ekman number, showing satisfactory quantitative agreement between the modified timedependent solution and the numerical simulations.

\section{Introduction}

Motivated by the wish to understand the fundamental dynamics of rotating fluids in planetary interiors and atmospheres, flows in rotating fluid-filled containers have been studied extensively for a very long time (for example Kelvin 1880; Greenspan 1964; Kudlick 1966; Wedemeyer 1966; Greenspan 1968; Malkus 1989; Hollerbach \& Kerswell 1995; Kerswell \& Barenghi 1995; Kerswell 1999). Motions in contained rotating fluids can be largely divided into two different categories: unforced initialvalue problems and forced problems. The initial-value problem describes how an initial state of the prescribed fluid motion, affected by both the viscous dissipation and rotation, decays over the time scale $O\left(E^{-1 / 2}\right)$, where the Ekman number $E$ is asymptotically small, toward a state of rigid-body rotation, the natural final state of a uniformly rotating viscous fluid (see, for example, Greenspan 1964; Kudlick 1966; Greenspan 1968). This paper is primarily concerned with asymptotic and numerical solutions of the initial-value problem in a rapidly rotating circular cylinder with an arbitrarily small but fixed $E$ subject to any physically acceptable initial condition. Since it is difficult to accurately measure time-dependent solutions of the initial value problem in experimental studies in rapidly rotating systems (see, for example, Fultz 1959; Malkus 1989; Aldridge \& Stergiopoulos 1991; Manasseh 1992), asymptotic solutions of the initial-value problem in rapidly rotating systems can only be checked by fully numerical simulations of the same problem.

The analysis of an asymptotic time-dependent solution of the initial-value problem in a rotating cylinder consists of two major parts (see, for example, Greenspan 1968): (i) derive the viscous decay factors for given inertial modes and (ii) obtain the 
asymptotic time-dependent solution on the basis of the decay factors and the inertial modes. A number of authors (for example Kudlick 1966; Wedemeyer 1966; Greenspan 1968; Kerswell \& Barenghi 1995; Kerswell 1999) have carried out the first part of the analysis. In an important study estimating the viscous decay rates for a rotating circular cylinder, Kerswell \& Barenghi (1995) compared the two existing asymptotic results, obtained by Kudlick (1966) and Wedemeyer (1966) who treated the relevant surface integral differently, with the numerically calculated values of the decay factors for a number of inertial modes with frequencies $\omega=O(1)$. To understand the resonant collapse observed in experiments (see, for example, Manasseh 1992), Kerswell (1999) studied a triad resonance by considering a three-inertial-mode interaction in a rotating circular cylinder. In the appendix of that paper, he calculated the viscous decay factor for two inertial modes with frequencies $\omega=O(1)$, up to $E=10^{-8}$, in a rotating cylinder with the stress-free condition at the top and bottom surface, showing that adding the interior dissipation contribution is helpful in evaluating the viscous decay factor.

The second part of the analysis, deriving and checking asymptotic time-dependent solutions of the initial-value problem subject to physically acceptable initial conditions, has received relatively less attention. In this paper, we present a modified asymptotic expression which proves more accurate at an arbitrarily small but fixed $E$ compared to Greenspan's original asymptotic theory. An essential ingredient for this better accuracy is the inclusion of inertial waves having a wide spectrum of frequencies and wavelengths, which contribute at the next order in the expansion if the spatial scale of the initial flow is $O(1)$. It turns out that these higher-order terms are surprisingly significant at the small but finite $E$ commonly considered in experiments and numerical computations. To derive this modified expression, we have to evaluate the viscous decay factors not only for the fast oscillation modes with $\omega=O(1)$ but also the slow oscillation modes with, for example, $\omega=O\left(E^{1 / 5}\right)$ or $\omega=O\left(E^{1 / 3}\right)$. It follows that we require an analytical expression of the viscous decay factors that is valid for a broad range of inertial modes, the detail of which will be discussed further in $\S 4$. In contrast to the first part of the analysis, a comparison between asymptotic solutions of the initial-value problem and the corresponding numerical solutions has not been carried out for a rapidly rotating fluid-filled cylinder.

We revisit the initial-value problem in a rotating cylindrical container by carrying out four different but related analyses. First, we derive a modified asymptotic expression for the viscous decay factors that is valid for a broad range of inertial modes required for an asymptotic solution of the initial-value problem at an arbitrarily small but fixed $E$. We then perform a fully numerical analysis, showing satisfactory quantitative agreement between the viscous decay factors given by the modified asymptotic expression and the fuller numerics. With the asymptotic expression of the decay factors valid for a broad range of inertial modes, we derive a modified asymptotic solution of the initial-value problem in a rapidly rotating circular cylinder for an arbitrarily small but fixed $E$ subject to any physically acceptable initial condition. Finally, we perform numerical simulations of the initial value problem, showing satisfactory quantitative agreement between the modified time-dependent asymptotic solution and numerical integration of the governing equations, over the time scale $O\left(E^{-1 / 2}\right)$, starting from the same prescribed initial velocity distribution.

In what follows we shall begin by presenting the mathematical equations of the problems in $\S 2$. The existing asymptotic solution of the initial-value problem is briefly discussed in $\S 3$ and the modified asymptotic solution of the initial-value problem is derived and presented in $\S 4$. Numerical viscous decay factors and numerical simulations of the initial-value problem, as well as their comparison with 
the asymptotic solutions, are discussed in $\S 5$. The paper closes in $\S 6$ with a short summary and a few remarks.

\section{Mathematical formulation}

We consider an incompressible viscous fluid filling a circular cylinder of radius $s_{o}$ and length $d$, with aspect ratio $\Gamma=s_{o} / d$, rotating uniformly about its axis with a constant vertical angular velocity $\boldsymbol{\Omega}$. At a particular time, $t=0$, a physically acceptable initial condition consisting of a small-Rossby-number fluid motion, $\boldsymbol{U}_{0}$, is imposed within the rotating cylinder. The manner in which this initial state approaches the final state of solid-body rotation is described by the following dimensionless equations (Greenspan 1968) in the rotating reference frame:

$$
\begin{gathered}
\frac{\partial \boldsymbol{u}}{\partial t}+2 \hat{\boldsymbol{z}} \times \boldsymbol{u}=-\nabla p+E \nabla^{2} \boldsymbol{u}, \\
\nabla \cdot \boldsymbol{u}=0,
\end{gathered}
$$

subject to the boundary conditions

$$
\boldsymbol{u}(s=\Gamma, \phi, z)=\boldsymbol{u}(s, \phi, z=0)=\boldsymbol{u}(s, \phi, z=1)=0
$$

and the initial condition

$$
\boldsymbol{u}(s, \phi, z, t=0)=\boldsymbol{U}_{0}(s, \phi, z),
$$

where $\hat{z}$ denotes the unit vector parallel to the axis of rotation, $\boldsymbol{u}$ is the threedimensional velocity field, $\left(u_{s}, u_{\phi}, u_{z}\right)$ in cylindrical coordinates $(s, \phi, z)$ with the unit vectors $(\hat{\boldsymbol{s}}, \hat{\boldsymbol{\phi}}, \hat{z})$ and with $s=0$ representing the axis of rotation, and $p$ is the reduced pressure. In (2.1)-(2.2), the depth of the cylinder $d$ is used as the length scale and $\Omega^{-1}$ as the unit of time.

We shall discuss the existing asymptotic solution of this initial-value problem in $\S 3$, our modified asymptotic solution in $\S 4$ and our fully numerical solution of the same problem in $\S 5$.

\section{The existing solution of the initial-value problem}

A general account of the asymptotic solution for the initial-value problem in contained rotating systems for an asymptotically small Ekman number $E$ was given by Greenspan (1968). Prior to the discussion of our modified asymptotic solution and fully numerical solution, it is useful to describe briefly the existing asymptotic solution of the initial-value problem (Greenspan 1968).

The existing solution of the initial-value problem, when the initial state varies only slightly from rigid-body rotation, is obtained by the following steps. First, the fluid velocity $\boldsymbol{u}$ and other variables are expanded in half-powers of an asymptotically small but fixed $E$ (for example Greenspan 1968, equations 2.5.6 and 2.5.8),

$$
\begin{aligned}
\boldsymbol{u}=\boldsymbol{u}_{G}+\sum_{m n k} A_{m n k} \boldsymbol{u}_{m n k} \mathrm{e}^{\left[\mathrm{i} \omega_{m n k} t-E^{1 / 2}\left(d_{m n k}-\mathrm{i} \omega_{m n k 1}\right) t+\cdots\right]}+\tilde{\boldsymbol{u}}_{G}+\sum_{m n k} \tilde{\boldsymbol{u}}_{m n k} & \\
& +E^{1 / 2}\left(\sum_{m n k} \boldsymbol{u}_{m n k 1}+\sum_{m n k} \tilde{\boldsymbol{u}}_{m n k 1}+\cdots\right)+\cdots,
\end{aligned}
$$

where $E^{1 / 2}$ is an expansion parameter, $\boldsymbol{u}_{G}$ denotes the single interior geostrophic mode, $\boldsymbol{u}_{m n k}=O(1)$ represents an inviscid three-dimensional inertial mode described 
by the three indices $(m, n, k)$ which are the three spatial wavenumbers in the azimuthal, vertical and radial direction respectively (the explicit definition of $(m, n, k)$ is given below), $\omega_{m n k}$ denotes the frequency of the inviscid inertial mode, $\omega_{m n k 1}$ is the viscous correction of the inviscid frequency, $\tilde{\boldsymbol{u}}_{m n k}=O(1), \tilde{\boldsymbol{u}}_{G}=O(1)$ and $\tilde{\boldsymbol{u}}_{m n k 1}=O(1)$ are the boundary corrections due to the effect of viscosity, $d_{m n k}$ denotes the scaled viscous decay factor of the inertial mode, and $\boldsymbol{u}_{m n k 1}=O(1)$ represents the secondary interior solution. The pressure field can be expanded in a similar way. Since the single stationary geostrophic mode $\boldsymbol{u}_{G}$ and its viscous correction $\tilde{\boldsymbol{u}}_{G}$ are extensively discussed by Greenspan (1968) and since we are mainly concerned with oscillatory inertial modes and the corresponding oscillatory boundary layers, we shall not discuss the geostrophic mode further in detail.

Greenspan (1968, Section 2.5) gave a detailed description of the relevant physical processes taking place in the initial value problem. Direct viscous action is confined to a thin boundary layer at the container wall which produces a secondary interior circulation. For a given initial velocity distribution, viscosity eliminates this on the time scale $O\left(E^{-1 / 2}\right)$ and $E^{1 / 2}$ emerges as a significant expansion parameter which is used in the expansion (3.1) for the initial-value problem. He also noted, however, that the effects of spatial and temporal non-uniformities obscure the correct form of the expansion beyond the first viscous corrective $O\left(E^{1 / 2}\right)$ terms in the expansion (3.1).

The first step in solving the initial-value problem is to obtain the viscous decay factor, $d_{m n k}$, for a given inertial mode $\boldsymbol{u}_{m n k}$ for an arbitrarily small but fixed $E$. As carefully explained by Greenspan (1968), after substitution of the expansion (3.1) into the governing equations (2.1)-(2.2) and collection of the powers of $E^{1 / 2}$, three problems in the asymptotic sequence have to be solved in order to determine the viscous decay factors $d_{m n k}$ (for example Greenspan 1968, p. 42, Section 2.5). The first is for the zero-order interior solution of the inviscid motion, governed by the dimensionless equations (Greenspan 1968, p. 42, Section 2.5)

$$
\begin{gathered}
\mathrm{i} 2 \sigma_{m n k} \boldsymbol{u}_{m n k}+2 \hat{z} \times \boldsymbol{u}_{m n k}=-\nabla p_{m n k}, \\
\nabla \cdot \boldsymbol{u}_{m n k}=0,
\end{gathered}
$$

where $\omega_{m n k}=2 \sigma_{m n k}$ and $\left|\sigma_{m n k}\right|<1$. Non-axisymmetric solutions of the first problem can be written in the form

$$
\begin{gathered}
\hat{\boldsymbol{s}} \cdot \boldsymbol{u}_{m n k}=\frac{-\mathrm{i}}{2\left(1-\sigma_{m n k}^{2}\right)}\left[\frac{\sigma_{m n k} \eta}{\Gamma} J_{m-1}\left(\frac{\eta s}{\Gamma}\right)+\frac{m\left(1-\sigma_{m n k}\right)}{s} J_{m}\left(\frac{\eta s}{\Gamma}\right)\right] \\
\quad \times \cos (n \pi z) \mathrm{e}^{\mathrm{i}\left(m \phi+2 \sigma_{m n k} t\right)}, \\
\hat{\boldsymbol{\phi}} \cdot \boldsymbol{u}_{m n k}=\frac{1}{2\left(1-\sigma_{m n k}^{2}\right)}\left[\frac{\eta}{\Gamma} J_{m-1}\left(\frac{\eta s}{\Gamma}\right)-\frac{m\left(1-\sigma_{m n k}\right)}{s} J_{m}\left(\frac{\eta s}{\Gamma}\right)\right] \\
\quad \times \cos (n \pi z) \mathrm{e}^{\mathrm{i}\left(m \phi+2 \sigma_{m n k} t\right)}, \\
\hat{z} \cdot \boldsymbol{u}_{m n k}=\frac{-\mathrm{i} n \pi}{2 \sigma_{m n k}}\left[J_{m}\left(\frac{\eta s}{\Gamma}\right)\right] \sin (n \pi z) \mathrm{e}^{\mathrm{i}\left(m \phi+2 \sigma_{m n k} t\right)},
\end{gathered}
$$

where $J_{m}(x)$ denotes the standard Bessel function, $m$ denotes the azimuthal wavenumber assumed to be positive, $n$ represents the vertical wavenumber and $\eta$ is associated with the radial wavenumber $k$ and is determined by solutions of the transcendental equation

$$
\frac{\mathrm{d} J_{m}(\eta)}{\mathrm{d} \eta}+\frac{m \sigma_{m n k}}{\eta\left|\sigma_{m n k}\right|}\left[1+\left(\frac{\eta}{\Gamma n \pi}\right)^{2}\right]^{1 / 2} J_{m}(\eta)=0
$$


for given $m$ and $n$. Solutions $\eta$ to (3.7) can be arranged, with the aid of subscript notion, according to their sizes

$$
0<\eta_{m n 1}<\eta_{m n 2}<\eta_{m n 3}, \ldots,<\eta_{m n k}<\ldots,
$$

where $\eta_{m n k}$ denotes the $k$ th smallest solution to (3.7) for given $n$ and $m$. The relation between $\eta_{m n k}$ and $\sigma_{m n k}$ is given by

$$
\sigma_{m n k}= \pm\left[1+\left(\frac{\eta_{m n k}}{\Gamma n \pi}\right)^{2}\right]^{-1 / 2} .
$$

The axisymmetric solution $(m=0)$ can be expressed in a similar way (Greenspan 1968).

In the second problem, the viscous boundary layer correction is made to ensure that the tangential velocity vanishes at the bounding surface of the container (Greenspan 1968, p. 42, Section 2.5)

$$
\begin{gathered}
\mathrm{i} \omega_{m n k} \tilde{\boldsymbol{u}}_{m n k}+2 \hat{z} \times \tilde{\boldsymbol{u}}_{m n k}-\boldsymbol{n} \cdot \nabla \tilde{\boldsymbol{p}}_{m n k}=\frac{\partial^{2} \tilde{\boldsymbol{u}}_{m n k}}{\partial \xi^{2}}, \\
\frac{\partial\left(\boldsymbol{n} \cdot \tilde{\boldsymbol{u}}_{m n k 1}\right)}{\partial \xi}=\boldsymbol{n} \cdot \nabla \times\left(\boldsymbol{n} \times \tilde{\boldsymbol{u}}_{m n k}\right),
\end{gathered}
$$

where $\xi$ is a stretched boundary-layer coordinate and $\boldsymbol{n}$ denotes the normal vector of the bounding surface of the fluid container. The third problem in the asymptotic sequence after collecting the $O\left(E^{1 / 2}\right)$ terms, which determines the viscous decay factors, is given by (Greenspan 1968, p. 43, Section 2.5)

$$
\begin{gathered}
\mathrm{i} \omega_{m n k} \boldsymbol{u}_{m n k 1}+2 \hat{z} \times \boldsymbol{u}_{m n k 1}+\nabla p_{m n k 1}=\left(\mathrm{i} \omega_{m n k 1}-d_{m n k}\right) \boldsymbol{u}_{m n k}, \\
\nabla \cdot \boldsymbol{u}_{m k n 1}=0,
\end{gathered}
$$

which is an inhomogeneous boundary-value problem requiring a solvability condition. After the first and second problems are solved subject to appropriate boundary conditions, the viscous decay factors, $d_{m n k}$, important parameters in the initial value problem, can be determined by the solvability requirement for (3.11). It is shown that the viscous decay factors are given by (Greenspan 1968, p. 57, 58, equations 2.9 .12 or 2.9.13),

$$
D_{m n k}=E^{1 / 2} \mathrm{~d}_{m n k}=-\left(\frac{\int_{S} p_{m n k}^{*}\left(\boldsymbol{n} \cdot \tilde{\boldsymbol{u}}_{m n k 1}\right) \mathrm{d} S}{\int_{V}\left|\boldsymbol{u}_{m n k}\right|^{2} \mathrm{~d} V}\right) E^{1 / 2},
$$

where $D_{m n k}$ is the unscaled viscous decay factor, $p_{m n k}^{*}$ denotes the complex conjugate of the pressure field $p_{m n k}, \int_{V}$ represents a volume integral over the fluid container and $\int_{S}$ represents a surface integral over the bounding surface of the container.

With the asymptotic formula for the viscous decay factors $d_{m n k}$ given by (3.13), one can derive the general time-dependent solution of the initial-value problem. The values of the coefficients $A_{m n k}$ in (3.1) are obtained by a straightforward integration making use of the orthogonality relationship (Greenspan 1968, p. 59, equations 2.10.4)

$$
A_{m n k}=\frac{\int_{V} \boldsymbol{u}_{m n k}^{*} \cdot \boldsymbol{U}_{0} \mathrm{~d} V}{\int_{V}\left|\boldsymbol{u}_{m n k}\right|^{2} \mathrm{~d} V}
$$


where $\boldsymbol{u}_{m n k}^{*}$ denotes the complex conjugate of $\boldsymbol{u}_{m n k}$. With the availability of the mathematical expression for all inertial modes $\boldsymbol{u}_{m k n}$ (Greenspan 1968, p. 82), the general time-dependent solution of the initial-value problem to leading approximation is (Greenspan 1968, p. 58, equation 2.10.1)

$$
\boldsymbol{u}(\boldsymbol{r}, t)=\sum_{m n k}\left(\frac{\int_{V} \boldsymbol{u}_{m n k}^{*} \cdot \boldsymbol{U}_{0} \mathrm{~d} V}{\int_{V}\left|\boldsymbol{u}_{m n k}\right|^{2} \mathrm{~d} V}\right) \boldsymbol{u}_{m n k} \mathrm{e}^{\left(\mathrm{i} \omega_{m n k}-E^{1 / 2} d_{m n k}\right) t}
$$

for the ensuing transient motion over the time scale $O\left(E^{-1 / 2}\right)$. For the purpose of illustration without loss of essential physics, we have assumed an initial state of the prescribed fluid motion, $\boldsymbol{U}_{0}$, that does not possess mean circulation so that the single geostrophic mode can be disregarded. The existing asymptotic solution of the initial-value problem is given by (3.14) and (3.13).

It was pointed out that each inertial mode, which is separable into an inviscid component and a boundary-layer correction, breaks down when the effective modal wavelength is of the order $E^{1 / 2}$ (Greenspan 1968, p. 63 and 83). This implies that, for a given small $E$, there will be a cutoff in the inertial modes included in (3.14): the time scale $O\left(E^{-1 / 2}\right)$ corresponds to the cutoff frequency $\left|\omega_{m n k}\right|=O\left(E^{1 / 2}\right)$ while the spatial scale $O\left(E^{1 / 2}\right)$ gives rise to the cutoff radial wavenumber $k=O\left(E^{-1 / 2}\right)$. It follows that all the inertial modes having the range of frequencies

$$
O\left(E^{1 / 2}\right)<\left|\omega_{m n k}\right| \leqslant O(1),
$$

must be included in the asymptotic solution (3.14). Furthermore, the cutoff wavenumber means that all the inertial modes in the range of wavenumbers

$$
O(1) \leqslant k<O\left(E^{-1 / 2}\right),
$$

where $k$ is the radial wavenumber provided that $n=O(1)$ and $m=O(1)$, must be included in (3.14). Since the small frequency $\omega_{m n k}$ and the large wavenumber $k$ are related by $k \sim \omega_{m n k}^{-1}$ (Greenspan 1968), we only need to consider the range of the inertial modes restricted by (3.15). It should be also noticed that the time scale $O\left(E^{-1 / 2}\right)$ valid for an asymptotic solution of the initial value problem is fully consistent with the cutoff frequency and wavenumber of the asymptotic solution (3.14).

The existing asymptotic solution of the initial-value problem given by (3.14) is computed for a prescribed initial velocity distribution at a fixed small $E$ as a function of time and is then compared with direct numerical simulations of the same problem, which will be discussed in $\S 5$.

\section{A modified solution of the initial-value problem}

We emphasize that (i) we are concerned with time-dependent solutions of the initial-value problem over the time scale $O\left(E^{-1 / 2}\right)$, where $E$ is arbitrarily small but fixed, subject to any physically acceptable initial condition, and (ii) the value of $E$ determines the range of the inertial modes that must be included in an asymptotic solution of the initial-value problem. More explicitly, all the inertial modes whose frequencies $\omega_{m n k}$ satisfy

$$
\left|\omega_{m n k}\right|=O\left(E^{\beta}\right) \text { with } 0 \leqslant \beta<\frac{1}{2},
$$


must be included in an asymptotic solution of the initial value problem. Note that all the inertial modes defined by (4.1) have spatial scales larger than the cutoff scale $O\left(E^{1 / 2}\right)$ and have temporal scales shorter than the cutoff scale $O\left(E^{-1 / 2}\right)$.

We make the following observation: the effects of spatial and temporal nonuniformities obscure the form of the expansion even in the first viscous corrective terms in an asymptotic solution of the initial-value problem. This is because, for an inertial mode with $\beta \neq 0$ in (4.1), such as $\beta=1 / 5$, the thickness of the oscillatory viscous boundary layer is not of the order $E^{1 / 2}$ and the flux from the oscillatory viscous boundary layer is not of the order $E^{1 / 2}$. The fact that the boundary-layer thickness and flux strongly depend on $\beta$ suggests that we cannot take $E^{1 / 2}$ as an expansion parameter even for the first viscous corrective terms in an asymptotic solution of the initial-value problem.

This observation indicates that the expansion (3.1) of the asymptotic solution for an arbitrarily small but fixed $E$ and the corresponding three problems of the asymptotic sequence defined by (3.2)-(3.12) need to be modified, leading to the expansion

$$
\begin{aligned}
\boldsymbol{u}=\sum_{m n k} A_{m n k} \boldsymbol{u}_{m n k} \mathrm{e}^{\left[\mathrm{i} \omega_{m n k} t-\left(D_{m n k}-\mathrm{i} \omega_{m n k 1}\right) t\right]}+\sum_{m n k} \tilde{\boldsymbol{u}}_{m n k} & \\
& +\left(\sum_{m n k} \boldsymbol{u}_{m n k 1}+\sum_{m n k} \tilde{\boldsymbol{u}}_{m n k 1}\right),
\end{aligned}
$$

where $\left|\boldsymbol{u}_{m n k}\right|=O(1), \tilde{\boldsymbol{u}}_{m n k}=O(1),\left|\boldsymbol{u}_{m k n 1}\right| \ll 1$ and $\left|\tilde{\boldsymbol{u}}_{m n k 1}\right| \ll 1$. Note that boundarylayer solutions $\tilde{\boldsymbol{u}}_{m n k}$ are required because the interior inertial modes $\boldsymbol{u}_{m n k}$ given by (3.4)-(3.6) do not satisfy the non-slip boundary condition. It follows that all the corresponding boundary-layer corrections $\tilde{\boldsymbol{u}}_{m n k}$ must be included in the asymptotic solution of the initial-value problem. In the expansion (4.2), we have again assumed that the initial condition does not possess mean circulation,

$$
\int_{0}^{1} \int_{0}^{2 \pi} \hat{\boldsymbol{\phi}} \cdot \boldsymbol{U}_{0}(s, \phi, z) s \mathrm{~d} \phi \mathrm{d} z=0,
$$

so that the single geostrophic mode does not appear.

It is worth noting that the axisymmetric $\left(\boldsymbol{u}_{0 n k}\right.$ with $\left.m=0\right)$ and non-axisymmetric $\left(\boldsymbol{u}_{m n k}\right.$ with $\left.m>0\right)$ inertial modes require slightly different formulations in both the asymptotic and numerical analysis. For the purpose of clarity, we shall present the solutions for axisymmetric and non-axisymmetric modes separately. Substitution of (4.2) into the governing equations (2.1)-(2.2) also yields three problems in the asymptotic sequence. The first is the same as that defined by (3.2)-(3.3), describing the inviscid axisymmetric inertial modes $\boldsymbol{u}_{0 n k}$ or non-axisymmetric inertial modes $\boldsymbol{u}_{m n k}$ with half-frequencies $\sigma_{0 n k}$ and $\sigma_{m n k}$. The second problem in the asymptotic sequence, describing the oscillatory viscous boundary layer at the bounding surface of the cylinder, is also the same as that for the existing solution. It is straightforward to solve (3.9)-(3.10) to find $\tilde{\boldsymbol{u}}_{m n k}$ for the viscous boundary layer (see, for example, Kudlick 1966; Kerswell \& Barenghi 1995).

The third problem in the asymptotic sequence, concerning the first viscous corrective terms that determine the values of the viscous decay factors, is, according to the modified expansion (4.2), given by

$$
\begin{gathered}
2 \mathrm{i} \sigma_{m n k} \boldsymbol{u}_{m n k 1}+2 \hat{z} \times \boldsymbol{u}_{m n k 1}+\nabla p_{m n k 1}=\left(2 \mathrm{i} \sigma_{m n k 1}-D_{m n k}\right) \boldsymbol{u}_{m n k}+E \nabla^{2} \boldsymbol{u}_{m n k}, \\
\nabla \cdot \boldsymbol{u}_{m n k 1}=0,
\end{gathered}
$$




\begin{tabular}{ccccccc}
\hline$(m, n)$ & $k=1$ & $k=2$ & $k=3$ & $k=4$ & $k=5$ & $k=6$ \\
$(0,1)$ & $3.810 \times 10^{-1}$ & $2.196 \times 10^{-1}$ & $1.518 \times 10^{-1}$ & $1.161 \times 10^{-1}$ & $9.542 \times 10^{-2}$ & $8.023 \times 10^{-2}$ \\
$(1,1)$ & $5.000 \times 10^{-1}$ & $2.562 \times 10^{-1}$ & $1.704 \times 10^{-1}$ & $1.274 \times 10^{-1}$ & $1.017 \times 10^{-1}$ & $8.462 \times 10^{-2}$ \\
$(1,3)$ & $8.878 \times 10^{-1}$ & $6.446 \times 10^{-1}$ & $4.749 \times 10^{-1}$ & $3.689 \times 10^{-1}$ & $2.996 \times 10^{-1}$ & $2.514 \times 10^{-1}$ \\
$(2,1)$ & $3.353 \times 10^{-1}$ & $2.002 \times 10^{-1}$ & $1.429 \times 10^{-1}$ & $1.112 \times 10^{-1}$ & $9.098 \times 10^{-2}$ & $7.701 \times 10^{-2}$ \\
$(2,3)$ & $7.659 \times 10^{-1}$ & $5.480 \times 10^{-1}$ & $4.135 \times 10^{-1}$ & $3.288 \times 10^{-1}$ & $2.718 \times 10^{-1}$ & $2.312 \times 10^{-1}$
\end{tabular}

TABLE 1. Several typical positive $\sigma_{m n k}$ of inertial modes in a rotating cylinder with the aspect ratio $\Gamma=1 / 1.9898$.

where $m \geqslant 0$ and $\sigma_{m n k 1}$ denotes the small viscous correction to the inviscid frequency, which is of less significance. It is of importance to note that, in the framework of the modified asymptotic expansion (4.2), the term $E \nabla^{2} \boldsymbol{u}_{m n k}$ in (4.3), representing the interior viscous dissipation, must be retained.

The inhomogeneous differential equation (4.3) requires a solvability condition whose real part yields the modified asymptotic expression for the viscous decay factors $D_{m n k}$. For the axisymmetric inertial oscillation modes with $m=0$, we obtain from the solvability condition that

$$
\begin{aligned}
D_{0 n k}=\left(n^{2} \pi^{2}\right) & \left(\frac{E^{1 / 2}}{\sigma_{0 n k}}\right)^{2}+\left[\left(1-\sigma_{0 n k}^{2}\right) E\right]^{1 / 2} \\
& \times\left[\frac{\left|\sigma_{0 n k}\right|^{1 / 2}}{\Gamma}\left(1-\sigma_{0 n k}^{2}\right)^{1 / 2}+\left(1-\sigma_{0 n k}\right)^{3 / 2}+\left(1+\sigma_{0 n k}\right)^{3 / 2}\right],
\end{aligned}
$$

where $\sigma_{0 n k}$ are the half-frequencies of axisymmetric inertial modes in the range $O\left(E^{1 / 2}\right)<\sigma_{0 n k} \leqslant O(1)$ required for an asymptotic solution of the initial-value problem. Several positive half-frequencies for both axisymmetric and non-axisymmetric modes are shown in table 1 in a rotating cylinder with aspect ratio $\Gamma=1 / 1.9898$. We choose this particular $\Gamma$ because it was used in previous studies (for example Kerswell \& Barenghi 1995), making the comparison of our analysis with previous work easier.

In the modified asymptotic expression (4.5), the viscous contribution to the viscous decay factors $D_{0 n k}$ comprises three different parts if we assume that $n=O(1)$ and $\Gamma=O(1)$ :

(i) from the horizontal boundary layers $\sim\left[\left(1-\sigma_{0 n k}^{2}\right) E\right]^{1 / 2}$;

(ii) from the vertical boundary layer $\sim\left(\left|\sigma_{0 n k}\right| E\right)^{1 / 2}\left(1-\sigma_{0 n k}^{2}\right)$;

(iii) from the interior flow $\sim\left(\frac{E^{1 / 2}}{\sigma_{0 n k}}\right)^{2}$.

We examine three typical cases of $\beta$ for the purpose of illustration:

(a) Consider the inertial modes with $\beta=0$ in (4.1), i.e. $\sigma_{0 n k}=O(1)$, which are required for an asymptotic solution of the initial-value problem. The contribution from the interior is subdominant to the Ekman boundary contribution. In this case, the existing formula (3.13) gives a correct result provided that $E$ is sufficiently small (Kerswell 1999).

(b) Consider the inertial modes with $\beta=1 / 5$ in $(4.1)$, i.e. $\sigma_{0 n k}=O\left(E^{1 / 5}\right)$, which are also required for the solution of the initial-value problem. The contribution from part (iii), which is neglected in the existing formula (3.13), is $O\left(E^{3 / 5}\right)$ while the contribution from the vertical boundary layer is also $O\left(E^{3 / 5}\right)$. In this case, the existing formula (3.13) cannot give a correct result regardless of how small $E$ is.

(c) Consider the inertial modes with $\beta=1 / 3$ in (4.1), i.e. $\sigma_{0 n k}=O\left(E^{1 / 3}\right)$, which are also needed for the solution of the initial-value problem. The contribution from part 
(iii) is $O\left(E^{1 / 3}\right)$ while the contribution from the horizontal boundary layer is $O\left(E^{1 / 2}\right)$ and the contribution from the vertical boundary layer is $O\left(E^{2 / 3}\right)$. In this case, the existing formula (3.13) again cannot yield a correct result regardless of how small $E$ is.

Similarly, the solvability condition for the inhomogeneous perturbation equation (4.3) gives rise to a modified asymptotic expression for the viscous decay factors, $D_{m n k}$, for all the non-axisymmetric inertial modes,

$$
\begin{aligned}
D_{m n k}= & \left(n^{2} \pi^{2}\right)\left(\frac{E^{1 / 2}}{\sigma_{m n k}}\right)^{2}+\left[\frac{M^{2} / \Gamma}{(n \pi \Gamma)^{2}+m\left(m-\sigma_{m n k}\right)}\right]\left[\left|\sigma_{m n k}\right|\left(1-\sigma_{m n k}^{2}\right)^{2} E\right]^{1 / 2} \\
& +\left[\frac{\left(1-\sigma_{m n k}\right)^{1 / 2}\left[\left(1-\sigma_{m n k}\right) M^{2}-2 m \sigma_{m n k}\right]}{(n \pi \Gamma)^{2}+m\left(m-\sigma_{m n k}\right)}\right]\left[\left(1-\sigma_{m n k}^{2}\right) E\right]^{1 / 2} \\
& +\left[\frac{\left(1+\sigma_{m n k}\right)^{1 / 2}\left[\left(1+\sigma_{m n k}\right) M^{2}-2 m \sigma_{m n k}\right]}{(n \pi \Gamma)^{2}+m\left(m-\sigma_{m n k}\right)}\right]\left[\left(1-\sigma_{m n k}^{2}\right) E\right]^{1 / 2}
\end{aligned}
$$

where $m>0$ and $M^{2}=m^{2}+n^{2} \pi^{2} \Gamma^{2}$. With the availability of the viscous decay rates, given by (4.5) and (4.6), a modified asymptotic solution of the initial value problem in a rapidly rotating cylinder is given by

$$
\boldsymbol{u}(\boldsymbol{r}, t)=\sum_{m n k}\left(\frac{\int_{V} \boldsymbol{u}_{m n k}^{*} \cdot \boldsymbol{U}_{0} \mathrm{~d} V}{\int_{V}\left|\boldsymbol{u}_{m n k}\right|^{2} \mathrm{~d} V}\right) \boldsymbol{u}_{m n k} \mathrm{e}^{\left(2 \mathrm{i} \sigma_{m n k} t-D_{m n k} t\right)},
$$

which, similar to (3.14), should include all the inertial modes whose frequencies satisfy (4.1).

The modified asymptotic solution of the initial-value problem as a function of time, given by (4.6) and (4.7), is computed for a prescribed initial condition for a small Ekman number and is then compared with direct numerical simulations of the same problem, which will be discussed in $\S 5$.

\section{Numerical analysis: comparison with the asymptotic results}

As pointed out by Kerswell \& Barenghi (1995), the accuracy of the asymptotic analysis needs to be verified by independent fully numerical analysis because of the difficulties in experimental studies of rapidly rotating systems (see, for example, Gans 1970; Aurnou \& Olson 2001). We shall first consider the numerical estimate of the viscous decay factors, an eigenvalue problem, and then perform direct numerical simulations of the initial value problem. In the numerical analysis, axisymmetric and non-axisymmetric solutions are treated separately.

For an axisymmetric solution, a velocity vector satisfying equation (2.2) in cylindrical geometry can be expressed in the form

$$
\boldsymbol{u}=-\frac{\partial \bar{\Psi}}{\partial z} \hat{\boldsymbol{s}}+\bar{\Phi} \hat{\boldsymbol{\phi}}+\frac{1}{s} \frac{\partial(s \bar{\Psi})}{\partial s} \hat{z}
$$

In terms of $\bar{\Psi}$ and $\bar{\Phi}$, the no-slip velocity condition on the sidewall becomes

$$
\bar{\Psi}=\frac{\partial \bar{\Psi}}{\partial s}=\bar{\Phi}=0 \quad \text { at } \quad s=\Gamma,
$$


while the non-slip velocity condition on the top and bottom of the cylinder is imposed by

$$
\bar{\Psi}=\frac{\partial \bar{\Psi}}{\partial z}=\bar{\Phi}=0 \quad \text { at } \quad z=0,1 .
$$

Making use of the expression (5.1) and applying $\hat{\boldsymbol{\phi}} \cdot$ and $\hat{\boldsymbol{\phi}} \cdot \nabla \times$ onto (2.1), we derive two independent scalar equations for the two eigenvalues, the half-frequency $\sigma_{0 n k}$ and the viscous decay factor $D_{0 n k}$ of an axisymmetric inertial mode,

$$
\begin{gathered}
\left(2 \mathrm{i} \sigma_{0 n k}-D_{0 n k}\right) \bar{\Phi}-2 \frac{\partial \bar{\Psi}}{\partial z}=E\left(\frac{\partial^{2} \bar{\Phi}}{\partial z^{2}}+\frac{\partial^{2} \bar{\Phi}}{\partial s^{2}}+\frac{1}{s} \frac{\partial \bar{\Phi}}{\partial s}-\frac{\bar{\Phi}}{s^{2}}\right) \\
\left(2 \mathrm{i} \sigma_{0 n k}-D_{0 n k}\right)\left(\frac{\partial^{2} \bar{\Psi}}{\partial z^{2}}+\frac{\partial^{2} \bar{\Psi}}{\partial s^{2}}+\frac{1 \partial \bar{\Psi}}{s \partial s}-\frac{\bar{\Psi}}{s^{2}}\right)+2 \frac{\partial \bar{\Phi}}{\partial z} \\
=E\left(\frac{\partial^{2}}{\partial z^{2}}+\frac{\partial^{2}}{\partial s^{2}}+\frac{1}{s} \frac{\partial}{\partial s}-\frac{1}{s^{2}}\right)^{2} \bar{\Psi}
\end{gathered}
$$

The problem is solved numerically by using the Galerkin method in which the potential fields, $\bar{\Phi}$ and $\bar{\Psi}$, are expanded in terms of the standard Chebyshev functions $T_{j}(x)$

$$
\left.\begin{array}{l}
\bar{\Psi}=s(1-\hat{z})^{2}\left(1-\hat{z}^{2}\right)^{2}\left[\sum_{j=0}^{J} \sum_{l=0}^{L} \bar{\Psi}_{j l}^{n k} T_{j}(\hat{z}) T_{l}(\hat{z})\right] \mathrm{e}^{\left(\mathrm{i} 2 \sigma_{0 n k}-D_{0 n k}\right) t}, \\
\bar{\Phi}=s(1-\hat{z})\left(1-\hat{z}^{2}\right)\left[\sum_{j=0}^{J} \sum_{l=0}^{L} \bar{\Psi}_{j l}^{n k} T_{j}(\hat{z}) T_{l}(\hat{z})\right] \mathrm{e}^{\left(\mathrm{i} 2 \sigma_{0 n k}-D_{0 n k}\right) t},
\end{array}\right\}
$$

where $\bar{\Psi}_{j l}^{n k}$ and $\bar{\Phi}_{j l}^{n k}$ are complex coefficients which are independent of time, $\hat{z}=2 z-1$ and $\hat{s}=2 s / \Gamma-1$, and the factors $s$ and $(1-\hat{s})^{2}$ are imposed so that the expansions are regular at the rotation axis $s=0$ and the boundary conditions are satisfied automatically.

For the numerical analysis of non-axisymmetric modes, the velocity vector $\boldsymbol{u}$ satisfying equation (2.2) in cylindrical geometry can be expressed in terms of two scalar potentials $\Psi$ and $\Phi$ (Marqués 1990)

$$
\boldsymbol{u}=\frac{1}{s} \frac{\partial \Psi}{\partial \phi} \hat{\boldsymbol{s}}+\left(\frac{\partial \Phi}{\partial z}-\frac{\partial \Psi}{\partial s}\right) \hat{\boldsymbol{\phi}}-\frac{1}{s} \frac{\partial \Phi}{\partial \phi} \hat{z} .
$$

An important advantage of using (5.7) is that the two scalar potentials are decoupled in the velocity boundary condition on the whole bounding surface of the cylinder. In terms of $\Psi$ and $\Phi$, the non-slip velocity condition on the sidewall is imposed by

$$
\Psi=\frac{\partial \Psi}{\partial s}=\Phi=0 \quad \text { at } \quad s=\Gamma
$$

while the non-slip velocity condition on the top and bottom becomes

$$
\Psi=\frac{\partial \Phi}{\partial z}=\Phi=0 \quad \text { at } \quad z=0,1
$$

Making use of the expression (5.7) and applying $\hat{z} \cdot \nabla \times$ and $\hat{s} \cdot \nabla \times$ onto (2.1), we derive two independent scalar equations for the two eigenvalues, the half frequency 


$\begin{array}{cccc}J & L & \left(D_{231}, \sigma_{231}\right) \text { for } k=1 & \left(D_{236}, \sigma_{236}\right) \text { for } k=6 \\ 20 & 20 & \left(8.483 \times 10^{-3}, 0.7669\right) & \left(2.315 \times 10^{-2}, 0.2308\right) \\ 22 & 22 & \left(8.830 \times 10^{-3}, 0.7669\right) & \left(2.373 \times 10^{-2}, 0.2307\right) \\ 24 & 24 & \left(8.924 \times 10^{-3}, 0.7670\right) & \left(2.431 \times 10^{-2}, 0.2307\right) \\ 26 & 26 & \left(8.830 \times 10^{-3}, 0.7670\right) & \left(2.485 \times 10^{-2}, 0.2307\right) \\ 28 & 28 & \left(8.852 \times 10^{-3}, 0.7670\right) & \left(2.530 \times 10^{-2}, 0.2308\right) \\ 30 & 30 & \left(8.835 \times 10^{-3}, 0.7670\right) & \left(2.563 \times 10^{-2}, 0.2308\right) \\ 32 & 32 & \left(8.839 \times 10^{-3}, 0.7670\right) & \left(2.583 \times 10^{-2}, 0.2309\right) \\ 34 & 34 & \left(8.848 \times 10^{-3}, 0.7670\right) & \left(2.593 \times 10^{-2}, 0.2309\right) \\ 36 & 36 & \left(8.856 \times 10^{-3}, 0.7670\right) & \left(2.596 \times 10^{-2}, 0.2309\right) \\ 38 & 38 & \left(8.860 \times 10^{-3}, 0.7670\right) & \left(2.595 \times 10^{-2}, 0.2309\right) \\ 40 & 40 & \left(8.862 \times 10^{-3}, 0.7670\right) & \left(2.592 \times 10^{-2}, 0.2309\right)\end{array}$

TABLE 2. Numerical convergence of the viscous decay rates and the half-frequency $\sigma_{m n k}$ as a function of the truncation parameters $J$ and $L$ for $m=2$ and $n=3$ with two different values of $k$, for $E=10^{-5}$ and $\Gamma=1 / 1.9898$.

$\sigma_{m n k}$ and the decay factor $D_{m n k}$ of a non-axisymmetric inertial mode,

$$
\begin{array}{r}
\left(2 \mathrm{i} \sigma_{m n k}-D_{m n k}-E \nabla^{2}\right)\left[\frac{1}{s} \frac{\partial}{\partial s}\left(s \frac{\partial \Phi}{\partial z}\right)-\left(\nabla^{2}-\frac{\partial^{2}}{\partial z^{2}}\right) \Psi\right]+\frac{2}{s} \frac{\partial^{2} \Phi}{\partial z \partial \phi}=0, \\
{\left[2 \mathrm{i} \sigma_{m n k}-D_{m n k}-E\left(\nabla^{2}+\frac{2}{s} \frac{\partial}{\partial s}+\frac{1}{s^{2}}\right)\right]\left[\frac{\partial^{2} \Psi}{\partial s \partial z}-\left(\nabla^{2}-\frac{1}{s} \frac{\partial}{\partial s} s \frac{\partial}{\partial s}\right) \Phi\right]} \\
-\frac{2}{s} \frac{\partial^{2} \Psi}{\partial z \partial \phi}-\frac{2 E}{s}\left[\frac{1}{s} \frac{\partial}{\partial s}\left(s \frac{\partial^{2} \Phi}{\partial z^{2}}\right)-\left(\nabla^{2}-\frac{\partial^{2}}{\partial z^{2}}\right) \frac{\partial \Psi}{\partial z}\right]=0 .
\end{array}
$$

The numerical solutions for $\Phi$ and $\Psi$ are then expanded in the form

$$
\left.\begin{array}{l}
\Psi=s^{m}(1-\hat{z})^{2}\left(1-\hat{z}^{2}\right)\left[\sum_{j=0}^{J} \sum_{l=0}^{L} \Psi_{m j l}^{n k} T_{j}(\hat{z}) T_{l}(\hat{z})\right] \mathrm{e}^{\mathrm{i}\left(2 \sigma_{m n k} t+m \phi\right)-D_{m n k} t}, \\
\Phi=s^{m+1}(1-\hat{z})\left(1-\hat{z}^{2}\right)^{2}\left[\sum_{j=0}^{J} \sum_{l=0}^{L} \Phi_{m j l}^{n k} T_{j}(\hat{z}) T_{l}(\hat{z})\right] \mathrm{e}^{\mathrm{i}\left(2 \sigma_{m n k} t+m \phi\right)-D_{m n k} t},
\end{array}\right\}
$$

where $\Psi_{m j l}^{n k}$ and $\Phi_{m j l}^{n k}$ are complex coefficients independent of time, $m \geqslant 1$ and the factors $s^{m}$ and $s^{(m+1)}$ are imposed for regularity of the solution at $s=0$. Our numerical analysis has been carefully checked with that given by Kerswell \& Barenghi (1995). Several examples indicating the behaviour of numerical convergence of the solution are shown in table 2.

The primary purpose of our numerical analysis is to provide a valuable comparison with the results of the asymptotic analysis which is only valid for small values of the Ekman number $E \ll 1$. The main results of the numerical analysis for the viscous decay factors, together with those from the asymptotic analysis, are shown in tables 3 and 4.

We first discuss the viscous decay factors for the axisymmetric modes with $m=0$. Several typical values of the viscous decay factors are shown in table 3 for the six inertial modes $(m=0, n=1, k=1, \ldots, 6)$ with $E=10^{-4}$ and $E=10^{-5}$. It is of significance to notice that all the inertial modes shown in table 3 are needed in an asymptotic solution of the initial-value problem for $E \leqslant O\left(10^{-4}\right)$. For the purpose 
E

(A) $10^{-4} 3.001 \times 10^{-2}$

(B) $10^{-4} 3.681 \times 10^{-2}$

(C) $10^{-4} 3.767 \times 10^{-2}$

(A) $10^{-5} 9.489 \times 10^{-3}$

(B) $10^{-5} 1.017 \times 10^{-2}$

(C) $10^{-5}$

$$
k=2
$$

$k=3$

$k=4$

$2.874 \times 10^{-2}$

$4.921 \times 10^{-2}$

$5.042 \times 10^{-2}$

$9.088 \times 10^{-3}$

$1.114 \times 10^{-2}$

$1.130 \times 10^{-2}$
$2.755 \times 10^{-2} \quad 2.670 \times 10^{-2}$ $6.951 \times 10^{-2} \quad 9.797 \times 10^{-2}$ $7.142 \times 10^{-2} \quad 1.011 \times 10^{-1}$

$8.711 \times 10^{-3} \quad 8.442 \times 10^{-3}$

$1.291 \times 10^{-2} \quad 1.557 \times 10^{-2}$

$1.310 \times 10^{-2} \quad 1.581 \times 10^{-2}$

$$
k=5
$$$$
k=6
$$

$2.607 \times 10^{-2} \quad 2.558 \times 10^{-2}$ $1.345 \times 10^{-1} \quad 1.800 \times 10^{-1}$ $1.393 \times 10^{-1} \quad 1.857 \times 10^{-1}$ $8.243 \times 10^{-3} \quad 8.090 \times 10^{-3}$ $1.908 \times 10^{-2} \quad 2.342 \times 10^{-2}$ $1.937 \times 10^{-2} \quad 2.378 \times 10^{-2}$

TABLE 3. The viscous decay factor, $D_{0 n k}$, of the axisymmetric inertial modes with $(m=0, n=1)$ for various values of $k$ at $\Gamma=1 / 1.9898$ : (A) calculated from the existing formula (3.13) (Greenspan 1968, Equation 2.9.12 or 2.9.13), (B) computed from the modified asymptotic expression (4.5) and (C) from the fuller numerics.

$$
\begin{array}{ccccccc}
(\mathrm{m}=1, \mathrm{n}=1) & k=1 & k=2 & k=3 & k=4 & k=5 & k=6 \\
(\mathrm{~A}) & 9.128 \times 10^{-3} & 9.012 \times 10^{-3} & 8.637 \times 10^{-3} & 8.369 \times 10^{-3} & 8.174 \times 10^{-3} & 8.025 \times 10^{-3} \\
(\mathrm{~B}) & 9.523 \times 10^{-3} & 1.052 \times 10^{-2} & 1.204 \times 10^{-2} & 1.445 \times 10^{-2} & 1.771 \times 10^{-2} & 2.181 \times 10^{-2} \\
(\mathrm{C}) & 9.587 \times 10^{-3} & 1.063 \times 10^{-2} & 1.219 \times 10^{-2} & 1.464 \times 10^{-2} & 1.796 \times 10^{-2} & 2.212 \times 10^{-2} \\
& & & & & & \\
(\mathrm{~m}=1, \mathrm{n}=3) & k=1 & k=2 & k=3 & k=4 & k=5 & k=6 \\
\text { (A) } & 5.090 \times 10^{-3} & 8.549 \times 10^{-3} & 9.373 \times 10^{-3} & 9.450 \times 10^{-3} & 9.340 \times 10^{-3} & 9.191 \times 10^{-3} \\
\text { (B) } & 6.217 \times 10^{-3} & 1.069 \times 10^{-2} & 1.331 \times 10^{-2} & 1.598 \times 10^{-2} & 1.924 \times 10^{-2} & 2.324 \times 10^{-2} \\
\text { (C) } & 6.284 \times 10^{-3} & 1.077 \times 10^{-2} & 1.341 \times 10^{-2} & 1.612 \times 10^{-2} & 1.942 \times 10^{-2} & 2.347 \times 10^{-2} \\
& & & & & & \\
(\mathrm{~m}=2, \mathrm{n}=1) & k=1 & k=2 & k=3 & k=4 & k=5 & k=6 \\
\text { (A) } & 9.181 \times 10^{-3} & 8.773 \times 10^{-3} & 8.460 \times 10^{-3} & 8.239 \times 10^{-3} & 8.074 \times 10^{-3} & 7.945 \times 10^{-3} \\
\text { (B) } & 1.006 \times 10^{-2} & 1.127 \times 10^{-2} & 1.329 \times 10^{-2} & 1.623 \times 10^{-2} & 2.000 \times 10^{-2} & 2.459 \times 10^{-2} \\
\text { (C) } & 1.011 \times 10^{-2} & 1.133 \times 10^{-2} & 1.343 \times 10^{-2} & 1.641 \times 10^{-2} & 2.025 \times 10^{-2} & 2.491 \times 10^{-2} \\
& & & & & & \\
(\mathrm{~m}=2, \mathrm{n}=3) & k=1 & k=2 & k=3 & k=4 & k=5 & k=6 \\
\text { (A) } & 7.272 \times 10^{-3} & 9.115 \times 10^{-3} & 9.429 \times 10^{-3} & 9.375 \times 10^{-3} & 9.236 \times 10^{-3} & 9.086 \times 10^{-3} \\
\text { (B) } & 8.786 \times 10^{-3} & 1.207 \times 10^{-2} & 1.462 \times 10^{-2} & 1.759 \times 10^{-2} & 2.126 \times 10^{-2} & 2.570 \times 10^{-2} \\
\text { (C) } & 8.860 \times 10^{-3} & 1.215 \times 10^{-2} & 1.474 \times 10^{-2} & 1.775 \times 10^{-2} & 2.146 \times 10^{-2} & 2.595 \times 10^{-2}
\end{array}
$$

TABle 4 . The viscous decay factors of non-axisymmetric modes, $D_{m n k}$, for $\Gamma=1 / 1.9898$ at $E=10^{-5}$ : (A) calculated from the existing formula (Greenspan 1968, Equations 2.9.12 and 2.9.13), (B) the modified asymptotic expression (4.6) and (C) from the fuller numerics.

of comparison, we have calculated three different values of the viscous decay factor: (A) computed from the existing formula (3.13) (Greenspan 1968, Equation 2.9.12 or 2.9.13), (B) computed from our modified asymptotic expression (4.5) and (C) computed from the fully numerical analysis. It can be seen that the agreement between the modified asymptotic expression (4.5) and the fuller numerics is always within about $1 \%$ at $E=10^{-5}$ while the existing formula (3.13) can give rise to about $300 \%$ discrepancy. We look at two examples in detail. First, the mode $(m=0, n=1, k=1)$ with frequency $\omega_{011}=0.76$ (see table 1 ). In this case, the existing formula (3.13) gives a decay factor $D_{011}=9.49 \times 10^{-3}$, compared with $D_{011}=1.02 \times 10^{-2}$ from the modified expression (4.5) and $D_{011}=1.03 \times 10^{-2}$ from the fuller numerics. Second, we look at the mode $(m=0, n=1, k=6)$ with frequency $\omega_{016}=0.16$ (see table 1$)$. In this case, the existing formula (3.13) gives a decay factor $D_{016}=8.09 \times 10^{-3}$ while the 
modified expression (4.5) yields $D_{016}=2.34 \times 10^{-2}$ and the fuller numerics produces $D_{016}=2.38 \times 10^{-2}$.

Consider now the non-axisymmetric modes, with $m>0$. Table 4 shows several typical values of the viscous decay factor $D_{m n k}$ for two azimuthal wavenumbers $m=1$ and $m=2$, calculated from the existing formula (3.13), the modified expression (4.7) and full numerics at $E=10^{-5}$. Again all the inertial modes shown in table 4 are needed in an asymptotic solution of the initial-value problem for $E \leqslant O\left(10^{-4}\right)$. As expected, the behaviour of non-axisymmetric modes is largely the same as that of the axisymmetric modes. In general, the agreement between the modified asymptotic expression (4.6) and the fuller numerics is always within about $1 \%$ but the formula (3.13) can yield a discrepancy of about $300 \%$. Looking at two examples in detail, for the mode $(m=2, n=1, k=1)$ with frequency $\omega_{211}=0.68$ (see table 1$)$, the existing formula (3.13) gives a decay factor $D_{211}=9.18 \times 10^{-3}$, which should be compared to $D_{211}=1.01 \times 10^{-2}$ from the modified expression (4.6) and $D_{211}=1.01 \times 10^{-2}$ from the fuller numerics. For the mode $(m=2, n=1, k=6)$ with frequency $\omega_{216}=0.15$ (see table 1), the existing formula (3.13) gives a decay factor $D_{216}=7.95 \times 10^{-3}$ while the modified expression (4.6) yields $D_{216}=2.46 \times 10^{-2}$ and the full numerics produces $D_{216}=2.49 \times 10^{-2}$. The results show that our modified asymptotic expression can yield a satisfactory quantitative agreement with the fuller numerics for the inertial modes required for an asymptotic solution of the initial-value problem.

Finally, we consider time-dependent numerical solutions of the initial-value problem by carrying out numerical simulations up to the time scale $O\left(E^{-1 / 2}\right)$. Without loss of general physics in this linear problem, we shall assume an initial velocity distribution that contains only a single azimuthal wavenumber. For the axisymmetric solution, we integrate numerically the following two independent scalar equations with a given initial condition:

$$
\begin{gathered}
\frac{\partial \bar{\Phi}}{\partial t}-2 \frac{\partial \bar{\Psi}}{\partial z}=E\left(\frac{\partial^{2} \bar{\Phi}}{\partial z^{2}}+\frac{\partial^{2} \bar{\Phi}}{\partial s^{2}}+\frac{1}{s} \frac{\partial \bar{\Phi}}{\partial s}-\frac{\bar{\Phi}}{s^{2}}\right) \\
\frac{\partial}{\partial t}\left(\frac{\partial^{2} \bar{\Psi}}{\partial z^{2}}+\frac{\partial^{2} \bar{\Psi}}{\partial s^{2}}+\frac{1}{s} \frac{\partial \bar{\Psi}}{\partial s}-\frac{\bar{\Psi}}{s^{2}}\right)+2 \frac{\partial \bar{\Phi}}{\partial z}=E\left(\frac{\partial^{2}}{\partial z^{2}}+\frac{\partial^{2}}{\partial s^{2}}+\frac{1}{s} \frac{\partial}{\partial s}-\frac{1}{s^{2}}\right)^{2} \bar{\Psi}
\end{gathered}
$$

where we expand $\bar{\Psi}$ and $\bar{\Phi}$ in the form

$$
\left.\begin{array}{c}
\bar{\Psi}=s(1-\hat{z})^{2}\left(1-\hat{z}^{2}\right)^{2}\left[\sum_{n=0}^{N} \sum_{k=0}^{K} \bar{\Psi}_{n k}(t) T_{k}(\hat{z}) T_{n}(\hat{z})\right], \\
\bar{\Phi}=s(1-\hat{z})\left(1-\hat{z}^{2}\right)\left[\sum_{n=0}^{N} \sum_{k=0}^{K} \bar{\Psi}_{n k}(t) T_{k}(\hat{z}) T_{n}(\hat{z})\right],
\end{array}\right\}
$$

which satisfies the non-slip condition exactly on the bounding surface of the cylinder. Here $\bar{\Psi}_{n k}(t)$ and $\bar{\Phi}_{n k}(t)$ are real time-dependent coefficients. Equations (5.13)-(5.14) can be cast, after time discretization using an implicit scheme, in the form

$$
\begin{aligned}
\sum_{n=0}^{N} \sum_{k=0}^{K}\left[\left(2 \mathscr{S}_{\hat{n} \hat{k} n k}-\right.\right. & \left.\left.\mathscr{W}_{\hat{n} \hat{k} n k} \Delta t\right) \bar{\Phi}_{n k}^{j+1}-\mathscr{T}_{\hat{n} \hat{k} n k} \bar{\Psi}_{n k}^{j+1} \Delta t\right] \\
& =\sum_{n=0}^{N} \sum_{k=0}^{K}\left[\left(2 \mathscr{S}_{\hat{n} \hat{k} n k}+\mathscr{W}_{\hat{n} \hat{k} n k} \Delta t\right) \bar{\Phi}_{n k}^{j}+\mathscr{T}_{\hat{n} \hat{k} n k} \bar{\Psi}_{n k}^{j} \Delta t\right],
\end{aligned}
$$




$$
\begin{aligned}
\sum_{n=0}^{N} \sum_{k=0}^{K}\left[\left(2 \mathscr{X}_{\hat{n} \hat{k} n k}-\mathscr{Y}_{\hat{n} \hat{k} n k} \Delta t\right) \bar{\Psi}_{n k}^{j+1}-\mathscr{Z}_{\hat{n} \hat{k} n k} \bar{\Phi}_{n k}^{j+1} \Delta t\right] \\
=\sum_{n=0}^{N} \sum_{k=0}^{K}\left[\left(2 \mathscr{X}_{\hat{n} \hat{k} n k}+\mathscr{Y}_{\hat{n} \hat{k} n k} \Delta t\right) \bar{\Psi}_{n k}^{j}+\mathscr{Z}_{\hat{n} \hat{k} n k} \bar{\Phi}_{n k}^{j} \Delta t\right],
\end{aligned}
$$

where $\hat{n}=0,1, \ldots, N ; \hat{k}=0,1, \ldots, K, \bar{\Psi}_{n k}^{j+1}$ and $\bar{\Psi}_{n k}^{j}$ represent $\bar{\Psi}_{n k}$ at the time step $t=t_{j+1}$ and $t_{j}$, respectively, $\Delta t=t_{j+1}-t_{j}$, and the coefficients with four indexes, such as $\mathscr{T}_{\hat{n} \hat{k} n k}$ and $\mathscr{X}_{\hat{n} \hat{k} n k}$, denote complicated integrals involving four Chebyshev functions. Though any distribution of the initial velocity can be used in our numerical simulation, we will only discuss the numerical solutions taking the following parameterized initial distribution:

$$
\boldsymbol{U}_{0}=-\frac{\partial \bar{\Psi}_{K}}{\partial z} \hat{\boldsymbol{s}}+\bar{\Phi}_{K} \hat{\boldsymbol{\phi}}+\frac{1}{s} \frac{\partial\left(s \bar{\Psi}_{K}\right)}{\partial s} \hat{z}
$$

which represents a physically possible flow satisfying both $\nabla \cdot \boldsymbol{U}_{0}=0$ and the non-slip boundary condition at the cylinder wall, where

$$
\begin{gathered}
\bar{\Psi}_{K}=s(1-\hat{s})^{2}\left(1-\hat{z}^{2}\right)^{2} T_{K}(\hat{s}) T_{K}(\hat{z}), \\
\bar{\Phi}_{K}=s(1-\hat{s})\left(1-\hat{z}^{2}\right) T_{K}(\hat{s}) T_{K+1}(\hat{z}),
\end{gathered}
$$

and $T_{K}(x)$ denotes the standard Chebyshev function. The same initial condition is also used in calculating asymptotic solutions of the problem. We have also tried many different forms of the initial condition, all showing a broadly similar behaviour. In discussing solutions of the initial-value problem, we introduce the normalized kinetic energy, $E_{\text {kin }}$, of the time-dependent solution,

$$
E_{\text {kin }}(t)=\frac{\int_{0}^{2 \pi} \int_{0}^{1} \int_{0}^{\Gamma}|\boldsymbol{u}(s, z, \phi, t)|^{2} s \mathrm{~d} s \mathrm{~d} z \mathrm{~d} \phi}{\int_{0}^{2 \pi} \int_{0}^{1} \int_{0}^{\Gamma}\left|\boldsymbol{U}_{0}\right|^{2} s \mathrm{~d} s \mathrm{~d} z \mathrm{~d} \phi},
$$

where $\boldsymbol{u}$ represents the time-dependent velocity calculated either from direct numerical simulations or from the existing solution (3.14) or from the modified solution (4.6).

Three different axisymmetric solutions subject to exactly the same initial condition are shown in figure $1(a-c)$ for a rotating cylinder with $\Gamma=1 / 1.9898$ : $(A)$ the existing asymptotic solution given by (3.14), $(B)$ the modified asymptotic solution given by (4.7) and $(C)$ the fully numerical simulation given by (5.16)-(5.17). All our numerical integrations are performed up to $t=O\left(E^{-1 / 2}\right)=100$ for $E=10^{-4}$ and the values of $K$ in figures $1(a)-1(c)$ signify the scales of the initial velocity distributions which are, in all three cases, much larger than $O\left(E^{1 / 2}\right)$. Evidently, the modified asymptotic solution (4.7) of the initial-value problem shows a satisfactory agreement with the numerical simulation. It is worth noting that, in calculating the kinetic energy $E_{\text {kin }}$ for both the existing solution (3.14) and the modified solution (4.7), the contribution from the flow confined in the viscous boundary layer is not included. This exclusion may explain small but noticeable differences between the modified solution (4.7) and numerical simulations shown in figure 1.

For the numerical simulation with a non-axisymmetric initial condition, we integrate the following two independent scalar equations,

$$
\left(\frac{\partial}{\partial t}-E \nabla^{2}\right)\left[\frac{1}{s} \frac{\partial}{\partial s}\left(s \frac{\partial \Phi}{\partial z}\right)-\left(\nabla^{2}-\frac{\partial^{2}}{\partial z^{2}}\right) \Psi\right]+\frac{2}{s} \frac{\partial^{2} \Phi}{\partial z \partial \phi}=0
$$



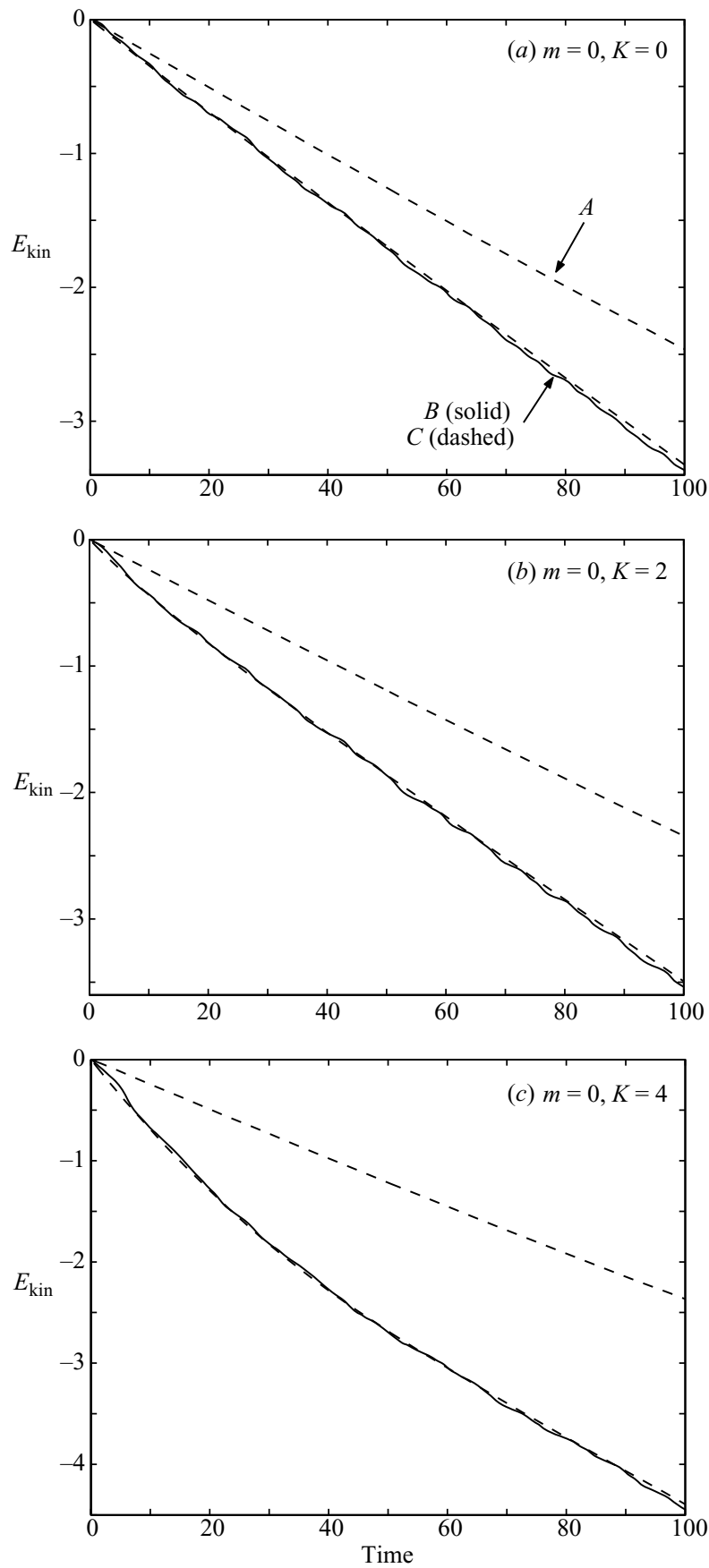

FIGURE 1. The normalized kinetic energy, $E_{\mathrm{kin}}(t)$, for solutions of the initial-value problem, in $a$ logarithmic scale, as a function of time with an axisymmetric initial velocity distribution at $E=10^{-4}$ for $\Gamma=1 / 1.9898$. Three different solutions with exactly the same initial condition are shown in each panel: $(A)$ the existing asymptotic solution given by $(3.14),(B)$ the modified asymptotic solution by (4.7) and $(C)$ fully numerical simulations from (5.16)-(5.17). 


$$
\begin{aligned}
{\left[\frac{\partial}{\partial t}-E\left(\nabla^{2}\right.\right.} & \left.\left.+\frac{2}{s} \frac{\partial}{\partial s}+\frac{1}{s^{2}}\right)\right]\left[\frac{\partial^{2} \Psi}{\partial s \partial z}-\left(\nabla^{2}-\frac{1}{s} \frac{\partial}{\partial s} s \frac{\partial}{\partial s}\right) \Phi\right] \\
& -\frac{2}{s} \frac{\partial^{2} \Psi}{\partial z \partial \phi}-\frac{2 E}{s}\left[\frac{1}{s} \frac{\partial}{\partial s}\left(s \frac{\partial^{2} \Phi}{\partial z^{2}}\right)-\left(\nabla^{2}-\frac{\partial^{2}}{\partial z^{2}}\right) \frac{\partial \Psi}{\partial z}\right]=0,
\end{aligned}
$$

where $\Phi$ and $\Psi$ are expanded in the form

$$
\left.\begin{array}{l}
\Psi=s^{m}(1-\hat{z})^{2}\left(1-\hat{z}^{2}\right)\left[\sum_{n=0}^{N} \sum_{k=0}^{K} \Psi_{m n k}(t) T_{k}(\hat{z}) T_{n}(\hat{z})\right] \mathrm{e}^{\mathrm{i} m \phi}, \\
\Phi=s^{m+1}(1-\hat{z})\left(1-\hat{z}^{2}\right)^{2}\left[\sum_{n=0}^{N} \sum_{k=0}^{K} \Phi_{m k n}(t) T_{k}(\hat{z}) T_{n}(\hat{z})\right] \mathrm{e}^{\mathrm{i} m \phi},
\end{array}\right\}
$$

which satisfies the non-slip condition exactly on the bounding surface of the cylinder, where $\Psi_{m n k}(t)$ and $\Phi_{m n k}(t)$ are time-dependent complex coefficients. Though any initial velocity distribution can be used, we have adopted the following prescribed parameterized distribution:

$$
\boldsymbol{U}_{0}=\operatorname{Re}\left[\frac{1}{s} \frac{\partial \Psi_{K}}{\partial \phi} \hat{\boldsymbol{s}}+\left(\frac{\partial \Phi_{K}}{\partial z}-\frac{\partial \Psi_{K}}{\partial s}\right) \hat{\boldsymbol{\phi}}-\frac{1}{s} \frac{\partial \Phi_{K}}{\partial \phi} \hat{z}\right]
$$

where

$$
\begin{aligned}
& \Psi_{K}=s^{m}\left[(1-\hat{z})^{2}\left(1-\hat{z}^{2}\right)^{2} T_{K}(\hat{z}) T_{K+1}(\hat{z})\right] \mathrm{e}^{\mathrm{i} m \phi}, \\
& \Phi_{K}=s^{m+1}\left[(1-\hat{z})\left(1-\hat{z}^{2}\right)^{2} T_{K}(\hat{z}) T_{K}(\hat{z})\right] \mathrm{e}^{\mathrm{i} m \phi},
\end{aligned}
$$

for both the asymptotic and numerical solutions of the initial value problem. Numerical integration is performed in a similar way to the axisymmetric solution.

Three different non-axisymmetric solutions subject to the same initial condition are shown in each panel in figure 2 again for a rotating cylinder with $\Gamma=1 / 1.9898$ : (A) the existing asymptotic solution given by (3.14), (B) the modified asymptotic solution given by (4.7) and (C) the fully numerical simulation. Again all our numerical integrations are performed up to $t=O\left(E^{-1 / 2}\right)=100$ for $E=10^{-4}$. The numerical solutions reveal a satisfactory agreement between the modified non-axisymmetric asymptotic solution (4.7) and the numerical simulation. Other forms of the nonaxisymmetric initial condition which we have used yield similar features to those shown in figure 2. Noticeable differences between the modified asymptotic solution (4.7) and the numerical solution in figure 2 are again attributable to the contribution from the viscous boundary-layer flow excluded in computing the kinetic energy of the asymptotic solution.

\section{Concluding remarks}

We have revisited the initial-value problem in a rapidly rotating circular fluid-filled cylinder that is characterized by an arbitrary small but fixed Ekman number $E$. We present solutions to four different but related problems. First, we derive a modified asymptotic expression for the viscous decay factors valid for the inertial modes of a broad range of frequencies that must be included in an asymptotic solution of the initial-value problem. We then perform a fully numerical analysis verifying the accuracy of the asymptotic viscous decay factors for a wide spectrum of the inertial modes. On the basis of the modified expression, we derive a modified asymptotic solution of the initial-value problem in a rapidly rotating circular cylinder. Finally, we perform numerical simulation, showing satisfactory quantitative agreement between 

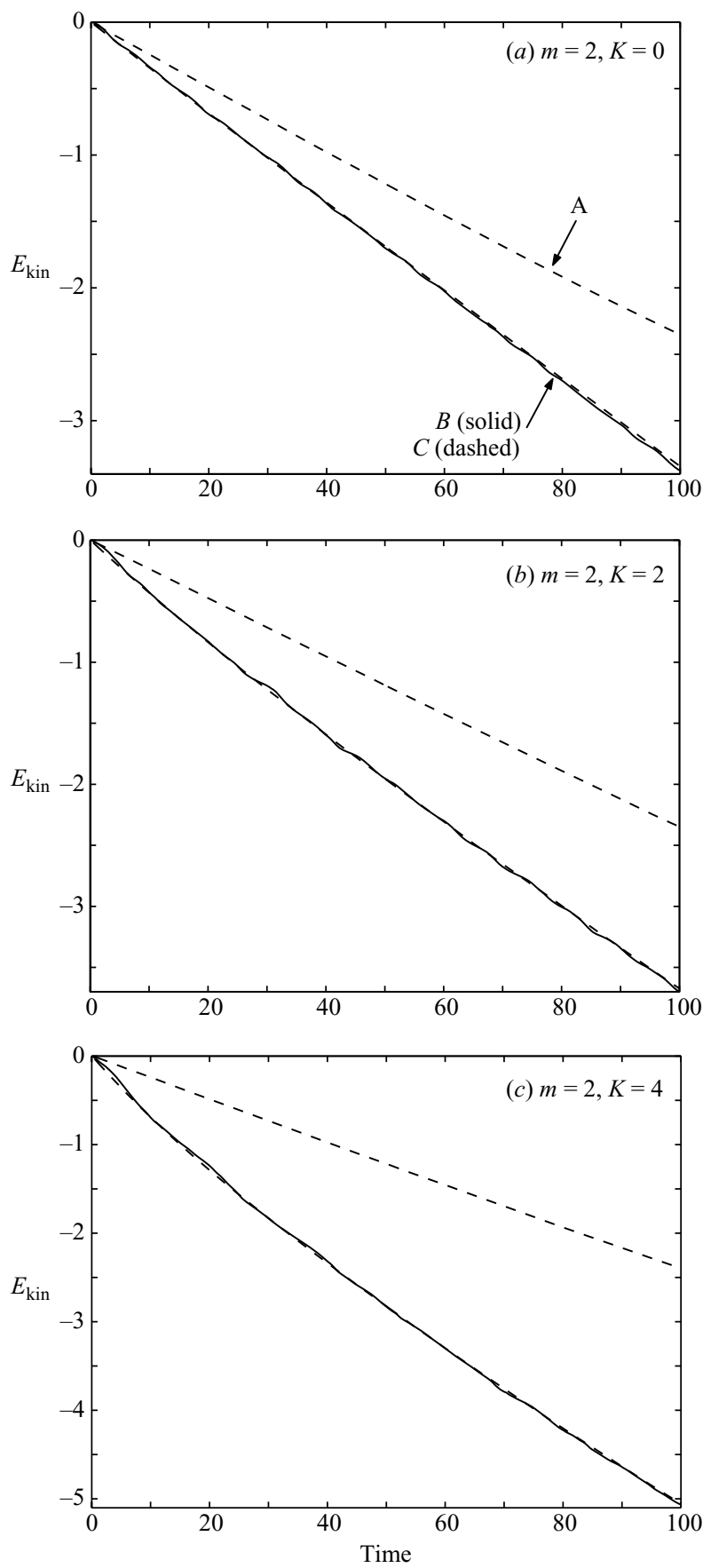

FIGURE 2. The normalized kinetic energy, $E_{\mathrm{kin}}(t)$, for solutions of the initial-value problem, in $a$ logarithmic scale, as a function of time with the $m=2$ initial velocity distribution at $E=10^{-4}$ for $\Gamma=1 / 1.9898$. Three different solutions with exactly the same initial condition are shown in each panel: $(A)$ the existing asymptotic solution $(3.14),(B)$ the modified asymptotic solution $(4.7)$ and $(C)$ fully numerical simulations. 
the modified asymptotic solution of the the initial-value problem and the fully numerical simulation.

This work represents, to the best knowledge of the authors, the first attempt to compare an asymptotic time-dependent solution of the initial-value problem with the corresponding solution obtained by numerical simulation in a rapidly rotating cylinder. The results of our modified asymptotic and numerical analyses suggest that the effects of spatial and temporal non-uniformities obscure the form of the expansion even in the first viscous corrective terms for an asymptotic solution at an arbitrarily small but fixed $E$ and, consequently, that $E^{1 / 2}$ cannot be taken as an expansion parameter even for the first viscous corrective terms in the process of deriving the time-dependent asymptotic solution for small but fixed $E$. It is worth mentioning that the spatial and temporal non-uniformity in the initial-value problem is, in many ways, similar to that found in the problem of convective instabilities for moderately small Prandtl numbers (Zhang, Liao \& Busse 2007).

In the present analysis, nonlinear effects, both in the interior and the boundary layers, are completely neglected. If the Rossby number $R o$ is sufficiently small, $R o \ll O\left(E^{1 / 2}\right)$, it is expected that the nonlinear effects are unlikely to play an important role in the initial-value problem. However, the nonlinear effects would become significant when $R o \geqslant O\left(E^{1 / 2}\right)$. An important question concerning how the nonlinear effects at a moderately small Rossby number become more important than the additional terms of the linear theory is beyond the scope of this study.

Finally, it is important to emphasize that the modified asymptotic solution of the initial-value problem given by (4.5)-(4.7) is valid for a rotating fluid cylinder that has arbitrarily small but fixed $E$ and is subject to any physically acceptable initial condition with a sufficiently small Rossby number. In this case, the size of $E$ determines the range of the inertial modes that should be included in the asymptotic expansion, requiring an analytical expression of the viscous decay factors valid for a broad range of inertial modes. If we consider an ideal mathematical problem by fixing the initial condition and, then, taking the limit $E \rightarrow 0$, the modified asymptotic solution (4.5)-(4.7) would converge to the existing asymptotic solution (3.13)-(3.14). This is because the inertial modes with $\omega=O(1)$ and $O(1)$ length scales will increasingly dominate the asymptotic expansion as $E \rightarrow 0$ with the fixed initial condition. But the modified asymptotic solution valid for small but finite $E$ with non-fixed initial conditions is needed in the practical physical application.

K. Z. would like to acknowledge helpful discussions with Professors F. H. Busse and R. R. Kerswell. We are also grateful to an anonymous referee who has offered many constructive comments. K.Z. is supported by UK PPARC and NERC grants while X. L. is supported by NSFC/10633030. The numerical computation is supported by Shanghai Supercomputer Center.

\section{REFERENCES}

Aldridge, K. D. \& Stergiopoulos, S. 1991 A technique for direct measurement of time-dependent complex eigenfrequencies of waves in fluids. Phys. Fluids A 3, 316-327.

Aurnou, J. M. \& Olson, P. L. 2001 Experiments on Rayleigh-Bénard convection, magnetoconvection and rotating magnetoconvection in liquid gallium. J. Fluid Mech. 430, 283-307.

FulTz, D. 1959 A note on overstability, and the elastoid-inertia oscillations of Kelvin, Solberg and Bjerknes. J. Met. 16, 199-208.

Gans, R. F. 1970 On the precession of a resonant cylinder. J. Fluid Mech. 41, 865-872. 
Greenspan, H. P. 1964 On the transient motion of a contained rotating fluid. J. Fluid Mech. 21, $147-154$.

Greenspan, H. P. 1968 The Theory of Rotating Fluids. Cambridge University Press.

Hollerbach, R. \& Kerswell, R. R. 1995 Oscillatory internal shear layers in rotating and precessing flows. J. Fluid Mech. 298, 327-339.

Kelvin, LoRd 1880 Vibrations of a columnar vortex. Phil. Mag. 10, 155-168.

KERSWELL, R. R. 1999 Secondary instabilities in rapidly rotating fluids: inertial wave breakdown. J. Fluid Mech. 382, 283-306.

Kerswell, R. R. \& BARENGHI, C. F. 1995 On the viscous decay-rates of inertial waves in rotating circular cylinder. J. Fluid Mech. 285, 215-247.

KudLick, M. D. 1966 On transient motions in a contained, rotating fluid. PhD Thesis, Mathematics Department MIT, USA.

MaLKus, W. V. R. 1989 An experimental study of the global instabilities due to the tidal (elliptical) distortion of a rotating elastic cylinder. Geophys. Astrophys. Fluid Dyn. 48, 123-134.

ManasseH, R. 1992 Breakdown regimes of inertia waves in a precessing cylinder. J. Fluid Mech. 243, 261-296.

MARQuÉs, F. 1990 On boundary conditions for velocity potentials in confined flows: Application to Couette flow. Phys. Fluids A 2, 729-737.

WedEMEYer, E. H. 1966 Viscous corrections to Stewartson's stability criterion. BRL Rep. 1325 US Army Ballistic Research Laboratory, Aberdeen Proving Ground, Maryland AD489687.

Zhang, K., Liao, X. \& Busse, F. H. 2007 Asymptotic theory of inertial convection in a rotating cylinder. J. Fluid Mech. 575, 449-471. 